\title{
GABA-Immunoreactivity in Inhibitory Motor Neurons of the Nematode Ascaris
}

\author{
Carl D. Johnson and Antony O. W. Stretton \\ Department of Zoology, University of Wisconsin, Madison, Wisconsin 53706
}

\begin{abstract}
We have used GABA-specific antisera to detect GABA-immunoreactivity in the motor neurons of the ventral nerve cord of Ascaris. We find that a subset of the individually identifiable commissures of motor neurons is specifically stained. On the basis of the location and morphology of stained commissures and of the location of stained cell bodies in the ventral nerve cord, we conclude that the labeled neurons comprise all members of the VI (inhibiting ventral muscle; 13 cells) and DI (inhibiting dorsal muscle; 6 cells) classes of inhibitory motor neurons (Stretton et al., 1978; Walrond et al., 1985). This result supports previous suggestions (e.g., del Castillo et al., 1964b) that GABA is the neurotransmitter released by the inhibitory motor neurons of nematodes.

In the anterior part of the animal, the inhibitory motor neuron commissures have small branches in the sublateral nerve cords that have not been previously described: VI commissures have dorsal sublateral branches, while DI cells have ventral branches. Posterior VI neurons have branches in the lateral nerve cords.
\end{abstract}

It is now more than 20 years since J. del Castillo and his colleagues suggested that 4-aminobutyric acid, commonly known as GABA, was an inhibitory neuromuscular transmitter in nematodes (del Castillo et al., 1964b). More recently, the motor neurons innervating body wall muscle cells have been studied anatomically in the free-living nematode Caenorhabditis elegans (White et al., 1976) and in the parasitic nematode Ascaris (Stretton et al., 1978). The number, arrangement, and morphology of identified cells are nearly identical in the 2 species; analogous cells between species are readily apparent. In addition, electrophysiological experiments in Ascaris have shown that 2 classes of motor neurons are inhibitory (Stretton et al., 1978; Walrond et al., 1985), one inhibiting dorsal muscle (DI motor neurons), the other inhibiting ventral muscle (VI motor neurons). However, so far, there has been little additional support for the proposal that GABA is a transmitter of the inhib-

Received Mar. 31, 1986; revised June 23, 1986; accepted June 24, 1986.

We thank G. E. Belzer and J. Guastella for assistance in performing some of the experiments, and J. Dallman, C. Hughes, and L. Vanderploeg for assistance in the preparation of the manuscript. We also thank Drs. S. Hoskin, T. Kingan, $R$. Wenthold, and J. Zempel for their generous gifts of anti-GABA sera. In addition we thank Dr. E. M. Hedgccock for suggesting the experiments, Dr. J. P. Walrond for his arrangement of and participation in the initial experiments and for his continuing interest, and Drs. J. Rand and J. White for their critical reading of the manuscript. This work was supported by United States Public Health Service Grant AI 20355.

Correspondence should be addressed to Carl D. Johnson, Zoology Research Bldg., 1117 W. Johnson St., Madison, WI 53706.

Copyright $\odot 1987$ Society for Neuroscience $0270-6474 / 87 / 010223-13 \$ 02.00 / 0$ itory motor neurons, partly because known antagonists of GABA action in other systems have minimal effects on inhibitory neuromuscular transmission in Ascaris. In order to provide further evidence for the role of GABA, we have undertaken a study of GABA localization in Ascaris motor neurons using immunocytochemical techniques.

The cell bodies of VI and DI motor neurons are located in the ventral cord (Fig. 1). Each cell has a process extending anteriorly and posteriorly in the ventral cord and a branch, called a commissure, that leaves the ventral cord to run circumferentially around the body to the dorsal cord. Members of the 3 classes of dorsal excitatory (DE) motor neurons also have commissures. In previous studies, we have shown by enzyme assays on dissected commissures that choline acetyltransferase (ChAT), the biosynthetic enzyme for acetylcholine, is concentrated in DE1, DE2, and DE3 motor neuron commissures (Johnson and Stretton, 1985). None of the VI and DI commissures examined contained ChAT. The present study shows that GABA-spccific antibodies stain the commissures of all VI and DI motor neurons and that the commissures of the $\mathrm{DE}$ motor neurons are not stained. These findings provide further evidence for the role of GABA as a transmitter of the inhibitory motor neurons in $A s$ caris. Further, we show that many of the VI and DI commissures have small branches in specific sublateral or lateral nerve cords. These observations raise new questions about the role of lateral interactions of the commissures in the neural control of nematode behavior.

\section{Materials and Methods}

Antisera. Anti-GABA sera were prepared from rabbits immunized with GABA conjugated to BSA with glutaraldehyde (Storm-Mathisen et al., 1983). The initial serum used for these experiments was supplied by $T$. Kingan and S. Hoskins from the Department of Biology, Columbia University; this serum had been purified by repeated passage over Sepharose-linked BSA-glutamate conjugate. Subsequent preparations, including those that were photographed for this paper, were labeled with anti-GABA sera supplied by R. Wenthold, now at the NIH, and J. Zempel from the Department of Neurophysiology, University of Wisconsin. These sera were purified by passage over Sepharose columns of BSA conjugates with glutamatc, aspartate, glutaminc, and glycine and their specificity demonstrated by dot-blots (Wenthold et al., 1986). We have also prepared 2 anti-GABA sera ourselves. In this case the sera were used without further purification. All of the sera specifically stained the same subset of motor neuron commissures. Background staining of non-neural structures was considerably higher when the unpurified sera were used.

Animals. Ascaris was obtained from the small intestine of pigs at the Jones Dairy Farm, Ft. Atkinson, WI. Animals used for these experiments were small- to medium-sized adult female and male worms (10$25 \mathrm{~cm}$ long).

Methods. Prior to labeling with the antiserum, the worm was injected with crude bacterial collagenase (Sigma C-9891), $4 \mathrm{mg} / \mathrm{ml}$ in Ascaris 


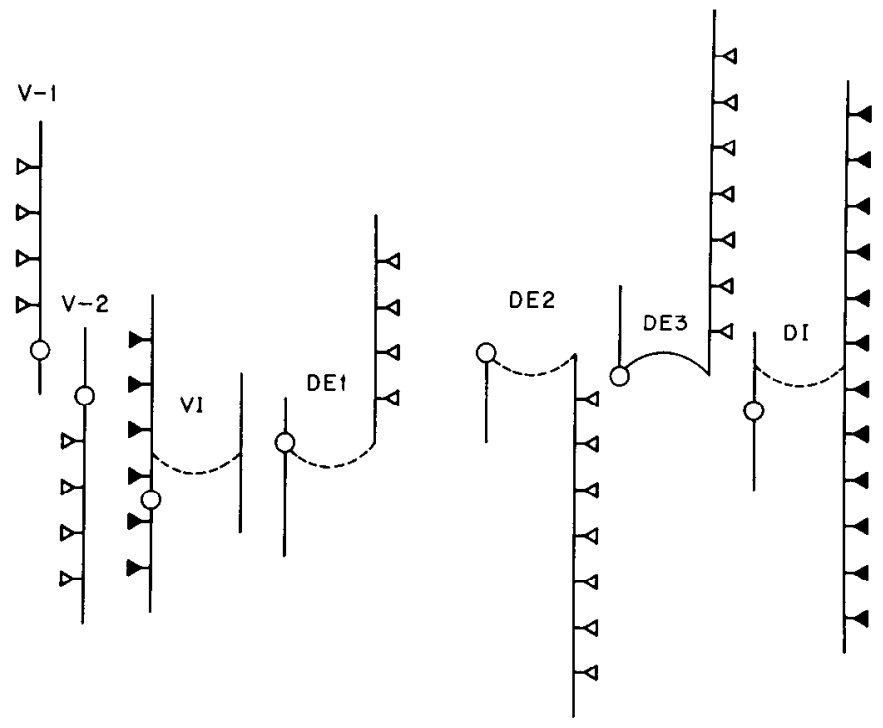

Figure 1. Diagrams showing representative shapes for 7 morphological classes of nematode motor neurons. Motor neuron cell bodies, located in the ventral nerve cord, are represented by circles. The vertical line extending from the cell body represents the process of the neuron in the ventral cord. $V-I$ and $V-2$ motor neurons are restricted to the ventral cord. The other 5 classes of cells (VI, DE1,DE2,DE3, and $D I)$ have a branch, the commissure, that exits from the ventral cord, turns either to the right or left, and runs circumferentially around the animal to the dorsal cord. The curved horizontal lines represent the commissures: dashed for right-handed commissures, solid for left-handed commissures. (The most common handedness for motor neurons of each type is shown. Some motor neurons have commissures of the opposite handedness.) Each of the 5 types of neurons with commissures also has a process in the dorsal cord, represented by a second vertical line. Attached triangles represent neuromuscular output zones. Open symbols indicate excitatory (or presumptive excitatory for $\mathrm{V}-1$ and V-2) output; filled symbols indicate inhibitory output (Stretton et al., 1978; Walrond et al., 1985).

saline (Stretton et al., 1978). In most cases, exposure to the collagenase was restricted to a portion of the worm by ligaturing the animal with string. The amount injected $(50-500 \mu \mathrm{l})$ depended on the size and length of worm ligatured. This treatment dissociates the muscle cells, which separate from the body wall to expose the neurons. After $1-2 \mathrm{hr}$ at $37^{\circ} \mathrm{C}$, the remaining body wall, which consists of the cuticle and the hypodermal layer in which the neurons are embedded, was cut along one lateral line, pinned out in a Sylgard-lined petri plate, washed free of dissociated tissues with $50 \mathrm{~mm}$ sodium phosphate, $\mathrm{pH} 7.4,150 \mathrm{~mm}$ $\mathrm{NaCl}$ (PBS), and immediately fixed by flooding the plate with $2 \%$ glutaraldehyde and $0.5 \%$ paraformaldehyde in PBS for $10-30 \mathrm{~min}$ at room temperature. After washing with PBS, the plate was flooded with $1 \%$ ethanolamine- $\mathrm{HCl}, \mathrm{pH} \mathrm{9}$, incubated at room temperature for $30 \mathrm{~min}$ (to block the remaining reactive glutaraldehyde sites), rinsed with PBS, and stored in $100 \mathrm{~mm}$ Tris, $\mathrm{pH} 8.0,300 \mathrm{mM} \mathrm{NaCl}$, and $1 \mathrm{mg} / \mathrm{ml} \mathrm{BSA}$ (Sigma) containing $0.05 \%$ Triton X-100 (TBS).

Before treatment with antibodies, the plate was rinsed with PBS and any tissue debris cleared from the Sylgard surface surrounding the worm pieces. In most cases, the tissue was treated with $1 \mathrm{mg} / \mathrm{ml}$ pronase (Calbiochem) in PBS for $30 \mathrm{~min}$ at room temperature, washed with PBS and then with TBS, and covered (50-100 $\mu$ l was sufficient to cover a 3 -cm-long piece of tissue) with TBS containing $10 \%$ calf serum and $0.5 \%$ Triton X-100 ( $\left.\mathrm{TBS}^{+}\right)$. The petri plates were placed in a moist container on a gentle rocker table for $12-24 \mathrm{hr}$ at $4^{\circ} \mathrm{C}$. After the TBS $^{+}$ was replaced with anti-GABA serum diluted (in most cases) $1 / 2000$ in TBS $^{+}$, the preparations were placed on the rocker table for 12-36 hr at $4^{\circ} \mathrm{C}$. Then unbound antibodies were removed by flooding the plate $6-$ 8 times with TBS over a period of $2 \mathrm{hr}$. The preparation was then treated with HRP-labeled goat anti-rabbit IgG (Bio-Rad, 170-6515) diluted $1 / 1000$ in $\mathrm{TBS}^{+}$. Incubation was generally overnight at $4^{\circ} \mathrm{C}$, after which the tissue was washed with TBS as above. Bound peroxidase was visualized by flooding the plate with $0.25 \mathrm{mg} / \mathrm{ml} \mathrm{4,4}$ '-diaminobenzidine in PBS containing $0.003 \% \mathrm{H}_{2} \mathrm{O}_{2}$. In some cases, the binding of antiGABA antibodies was detected by the 3-step peroxidase-antiperoxidase (PAP) technique (Storm-Mathisen et al., 1983). Staining was monitored with a dissecting microscope; it was generally allowed to proceed for 10 to $15 \mathrm{~min}$, after which the preparations were washed with PBS. Often preparations were collected over the course of several days, then treated with pronase and with antibodies, and stained in parallel.

Scoring the pattern of stained and unstained commissures was done initially with a dissecting microscope after mounting the preparations in glycerin jelly ( $8 \%$ gelatin in $50 \%$ glycerol). In some cases, the preparations were dehydrated through an alcohol series, cleared briefly in xylene, and mounted in Permount. Although the latter procedure has the advantage that staining of neural fine structures is clearer, its disadvantage is that the unstained commissures are generally invisible, whereas they can be readily seen in uncleared preparations.

In control experiments, either incubation with the primary antiserum was omitted or the primary antiserum was preincubated for $12 \mathrm{hr}$ at $4^{\circ} \mathrm{C}$ with $\mathrm{GABA}$-conjugated BSA or GABA-conjugated ovalbumin (Fig. 3). Specific staining of neurons was not observed in the control experiments.

Nomenclature. In total, there are 19 motor neuron commissures that stain with the anti-GABA sera. As will be shown below, 13 of the stained commissures appear to be from VI motor neurons, and 6 appear to be from DI cells. In what follows, the stained neurons are referred to by numbers (i.e., VI-1-VI-13 and DI-1-DI-6) that reflect their sequential position within the ventral nerve cord.

\section{Results}

We have performed experiments to localize the binding of GABA-specific antibodies within the nervous system of Ascaris. Of particular interest for this paper is the specific labeling of a subset of the motor neuron commissures and of motor neuron cell bodies in the ventral nerve cord.

\section{Staining of commissures}

The identity of the motor neuron connected to a specific commissure has, to a large extent, been determined previously by anatomical analysis of serial sections (Stretton et al., 1978), by the response of muscle cells to stimulation of single commissures (Stretton et al., 1978; Walrond et al., 1985), and by measurement of ChAT activity in dissected commissures (Johnson and Stretton, 1985). These experiments showed that each commissure has a number of distinctive morphological characteristics-position within the body, handedness (whether it traverses the right or left side of the animal), relative thickness, and whether or not it emerges directly from a cell body-that can be used to associate the commissure with a unique motor neuron. Forty of the 45 motor neurons that have commissures have been assigned to 1 of 5 morphological classes (VI, DE1, DE2, DE3, or DI; Fig. 1). Within most of the animal, members of these classes are organized in a sequentially repeated pattern. Each repeal contains 7 commissures arranged into 3 right-handed commissure pairs, followed by a single commissure to the left. Within the repeating unit, 2 of the right-handed pairs contain a DE1 and a VI motor neuron commissure, and the third contains a DE2 and a DI commissure. This latter group is close to the single left-handed commissure from a DE3 motor neuron. Along the body, there are 5 nearly identical repeats. (In the first repeat the first DE1/VI pair is left-handed and there is an extra right-handed (DE3) commissure located between the $2 \mathrm{DE} 1 / \mathrm{VI}$ pairs. In the fifth repeating unit, the second DE1/VI and the DE2/DI pairs often overlap.)

Figure 2 illustrates the intense, selective staining observed in members of 2 of the 5 classes of commissures when anti-GABA antibodies were detected with peroxidase-labeled second antibody, as described in Methods. Identical staining was observed 

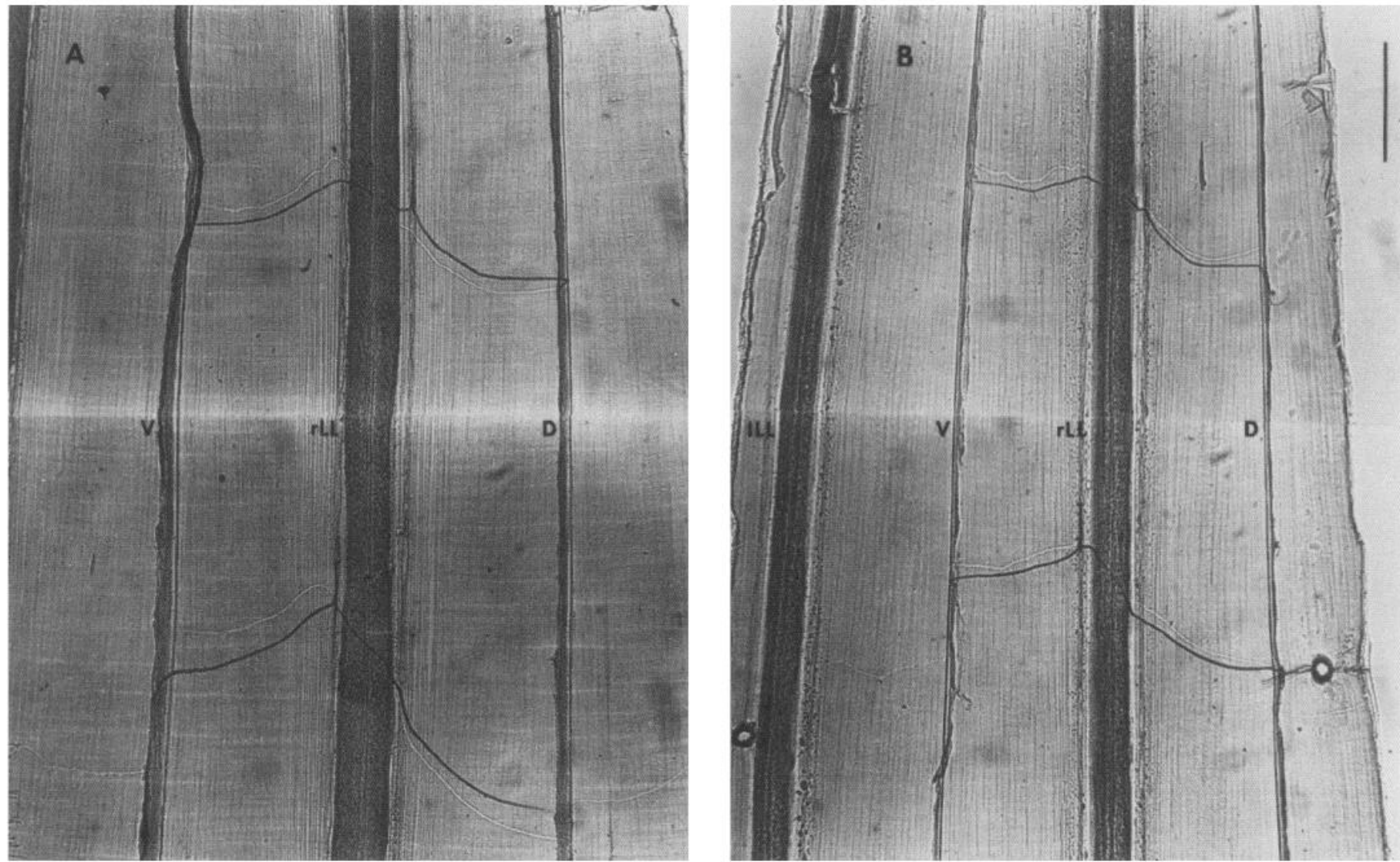

Figure 2. Photographs of a comparable region of the animal from 2 different preparations, stained with the anti-GABA antibodies. The region photographed includes the last 5 commissures in the first repeating unit. Commissures were assigned to specific motor neurons on the basis of position, handedness, and relative thickness. The more anterior right-handed commissure pair includes the unstained DE1 and the stained VI commissures (VI-3). The more posterior right-handed pair includes the unstained DE2 and the stained DI commissures (DI-2). The unstained lefthanded commissure is the DE3. For each right-handed pair, the stained commissure is posterior to the unstained commissure in the ventral hypodermis. In dorsal hypodermis, the stained commissure is anterior in $A$ and posterior in $B$. Ventral cord $(V)$ and dorsal cord $(D)$ are damaged in both of the preparations and are not stained. These preparations were mounted in glycerin jelly and photographed on a Zeiss Universal microscope using a $1 \times$ Olympus objective. Illumination was through a large-field condenser that was closed to its minimum diameter. Technical pan film 2415 (Kodak) was developed in $\mathrm{HCl} 10$ (Kodak) (dilution $\mathrm{F}, 6 \mathrm{~min}$ ) and printed through a No. 1 polycontrast filter (Kodak) to produce an especially low-contrast print. These procedures were followed in order to produce a print in which it is possible to visualize unstained commissures. Density in the lateral lines ( $r L L$, right lateral line; $l L L$, left lateral line) is artifactual, resulting from a small amount of (nonspecific) staining observed in these relatively thick structures and from density resultant from the use of a closed condenser. Scale bar, $1 \mathrm{~mm}$.

when anti-GABA antibodies were detected with the PAP technique. The photographs of comparable areas from 2 different preparations show a single stained commissure within the second and another stained commissure within the third righthanded pair of the first repeat. The other members of the righthanded pairs and the single left-handed commissure, representing the other 3 classes of commissures, are completely unstained and appear as clear lines on the grainy, hypodermal background. Commissure staining is specific. It is not observed when the primary antiserum is preadsorbed with GABA-conjugated BSA or GABA-conjugated ovalbumin (Fig. 3), but it is not blocked by preadsorption with glutamate, glutamine, aspartate, glycine, 3-aminobutyrate, or taurine conjugates (not shown).

Within each of the repeating units, there are 3 commissures that are stained with the anti-GABA sera, 1 in each of the (usually) right-handed pairs. On the basis of the following characteristics, the stained commissures are identified as belonging to the 2 VI motor neurons and the single DI cell of each repeat. First, the stained commissures emerge from ventral cord pro- cesses, not from cell bodies (Fig. 4). Previous studies have shown that commissures of the DE motor neurons emerge directly from their cell bodies, whereas those of the inhibitory motor neurons emerge from processes in the ventral cord (Fig. 1; Stretton et al., 1978). Second, within a commissure pair, the stained commissure is usually thinner than the unstained commissure. This distinction is clearest when comparing the ventral half of DE1 and VI commissures, although it sometimes fails to distinguish DE2 from DI ventrally. Generally the dorsal halves of excitatory and inhibitory commissures have the same diameter. Relative diameter has been used previously to distinguish between excitatory and inhibitory commissures (Johnson and Stretton, 1985). A third characteristic that we have quantified is the relative position of the stained commissure within the pair. From the observation of each of the stained commissures in 11-170 (average 80) animals (Fig. 5), we conclude that the stained commissure is, in each case, generally posterior to the unstained commissure as they traverse the hypodermis between the ventral cord and the lateral line. Within the 5 repeating units, there is a clear posterior bias to the positions of the stained commissures, 


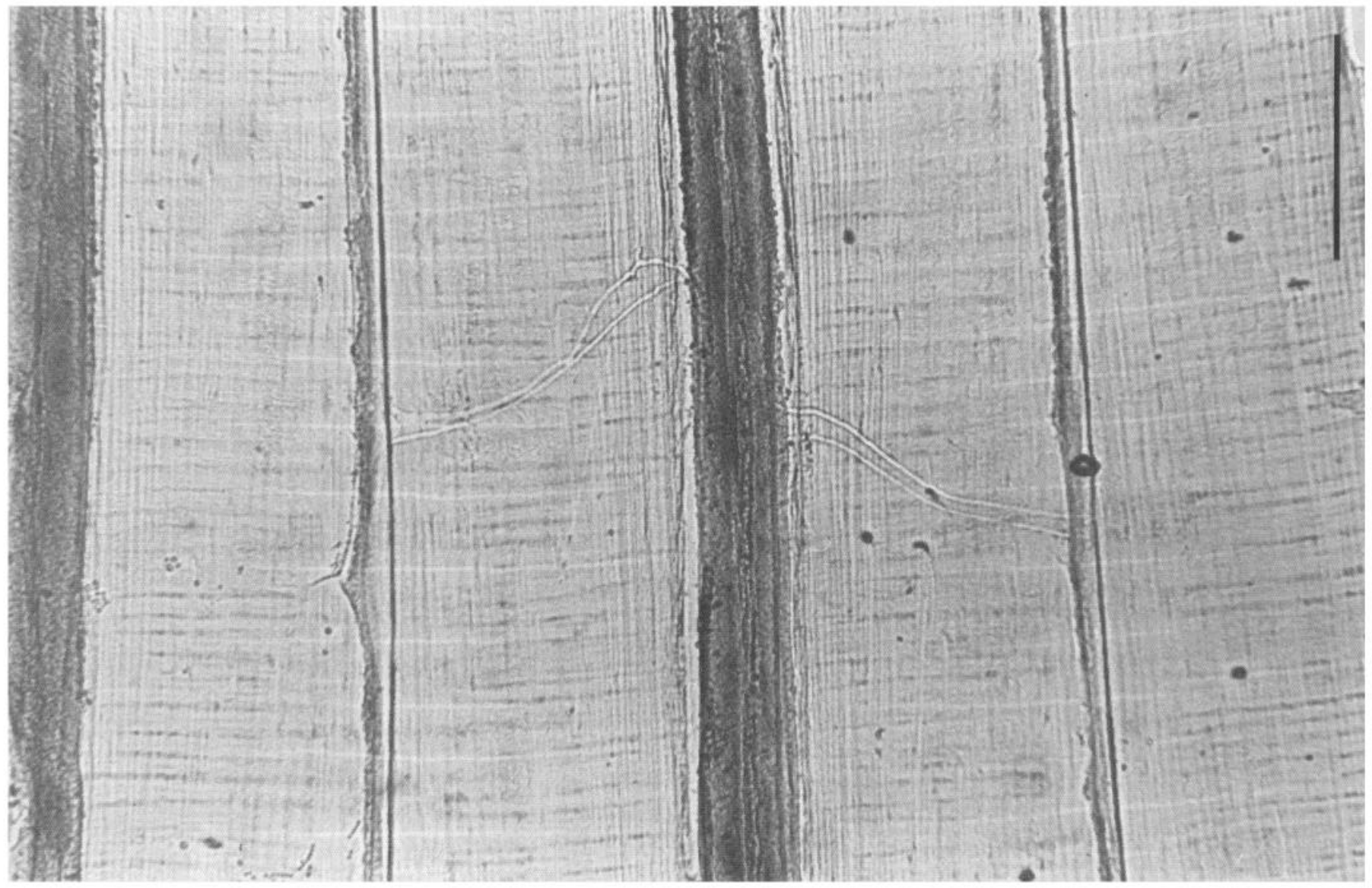

Figure 3. Photograph of DE1 and VI (VI-2) commissures stained with anti-GABA serum that had been incubated overnight with $2 \mu \mathrm{g} / \mathrm{ml}$ of GABA-ovalbumin conjugate before being applied to the preparation. Preadsorption with GABA conjugates blocks the specific staining of the inhibitory motor neuron commissure. Preparation was mounted, photographed, and the negative printed as described in Figure 2. Scale bar, $1 \mathrm{~mm}$.

although the extent of this bias varies for individual neurons. Thus the first VI commissure in repeating units 1-4 was posterior in $69,84,94$, and $80 \%$ of the preparations, respectively; the second VI commissure was posterior in $94,96,95$, and $95 \%$ of the preparations, respectively, and the DI commissure was posterior in $92,81,75$, and $67 \%$ of the preparations, respectively (Fig. 5). We have shown previously that ChAT activity is selectively localized in one of the commissures in right-handed pairs, and that there is a similar anterior bias to the position of the ChAT-positive commissure. Our conclusion, which is consistent with the present data, was that the generally anterior commissure in pairs belonged to a DE1 or a DE2 motor neuron (Johnson and Stretton, 1985).

Of the 6 motor neuron commissures anterior to the first repeating unit (see Fig. 6), 2 are stained with the anti-GABA sera. The most anterior stained commissure is part of a right-handed pair. The commissures of this pair are unusual in that they exit from the nerve cord at the top of the cord (medially) and traverse the region from ventral to right lateral line in the shelf of hypodermis that connects ventral and lateral ganglia (Goldschmidt, 1908); from lateral line to dorsal cord they are embedded in the hypodermis, which underlies the musculature that is the usual position for commissures. The next GABA-positive commissure is 1 of 3 left-handed commissures that exit the ventral cord (normally) just anterior to the first repeat. This stained commissure is generally posterior in ventral hypodermis (Fig. 5).

Posterior to the fifth repeat, there is a commissure pair to the left; the posterior commissure, which is extremely thin, is GABApositive (Figs. 5, 6). Finally, near the tail there is a single, righthanded commissure that is stained (Fig. 6). None of the 4 unidentified commissures in the tail is stained.

In total, of the 45 motor neuron commissures in female worms, 19 are stained with anti-GABA sera. The complete pattern of stained commissures is illustrated in Figure 6. The stained rightand left-handed commissures anterior to the first repeat are called VI-1 and DI-1, respectively. The commissures in the 5 repeating units are as follows: first repeat-VI-2, VI-3, DI-2; second repeat-VI-4, VI-5, DI-3; third repeat-VI-6, VI-7, DI4 ; fourth repeat-VI-8, VI-9, DI-5; and fifth repeat-VI-10, VI11, DI-6. The left-handed commissure posterior to the fifth repeat is called VI-12, and the right-handed commissure in the tail, VI-13.

The pattern of stained and unstained commissures observed in male worms is identical to that seen in females, except that in males there are 5 or 6 additional unstained, single, left-handed commissures, representing a class of male-specific motor neurons (A. O. W. Stretton, unpublished observations). One of these is located just posterior to the single right-handed commissure in the first repeat; the others are distributed posterior to the 
third repeat. In the bodies of worms of both sexes, there are also 8 very thin, unstained commissures ( 4 on each side of the animal), which extend from the lateral lines to the ventral nerve cord. These commissures are processes of neurons whose cell bodies are located in the lateral lines or in the tail. In male worms there are, in addition, a large number (ca. 75 on each side of the animal) of unstained lateral-to-ventral commissures associated with sensory papillae in the tail. Finally, there are lateral-to-ventral commissures associated with the nerve ring in the head that contain many fibers, some of which are stained. A detailed description of stained fibers and cell bodies in the head, the tail, and the pharynx will be presented in a separate publication.

The path of the motor neuron commissures is often deflected in an anterior or posterior direction as it approaches and passes through the lateral line. Within this area, the stained and unstained commissures often cross (e.g., Fig. $2 A$ ), such that the positional bias observed in ventral hypodermis is most often not seen dorsally (Fig. 5). Only 2 of the stained commissures, DI- 1 and VI-12, are generally posterior in dorsal hypodermis ( 72 and $93 \%$, respectively); the VI-1 commissure shows a $67 \%$ bias toward the anterior in dorsal hypodermis. For commissures within the 5 repeating units, however, the dorsal portion of the stained commissure was found anterior or posterior equally often (range, $39-62 \%$ posterior; average, $52 \%$ ).

\section{Staining of cell bodies}

Given the staining of commissures of the inhibitory motor neurons, we expected to observe corresponding stained cell bodies in the ventral nerve cord. Indeed, we have observed the presence of intensely stained cell bodies (e.g., Fig. 4) at 19 specific positions in the ventral cord. These positions are indicated in Figure 6. Each cell body has been observed in at least 5 preparations; cell bodies in the first repeat and further forward have been seen in 30-50 preparations. In these whole-mount preparations, it was not possible to trace stained fibers from their cell bodies to their commissures. However, since the number of stained cell bodies is exactly the same as the number of stained commissures, and since many of the stained cell bodies occur at positions in the ventral cord that are known from analysis of serial sections to contain the cell bodies of the inhibitory motor neurons (Stretton et al., 1978), we have concluded that they are the inhibitory motor neuron cell bodies. No other class of neuronal cell body located in the ventral cord was stained. Cell body staining was not found reliably in all preparations. This variability appears to have resulted from several factors. In many of the preparations the nerve cords were damaged prior to the application of antibodies. In adult Ascaris, the ventral and dorsal nerve cords are located at the medial surface of a thin hypodermal swelling, referred to as a hypodermal chalice (Fig. 9). Both the chalice and the nerve cord itself are fragile. Furthermore, intact nerve cords are almost completely surrounded by

Figure 4. Photographs of stained processes and cell bodies in the ventral nerve cord. $A$, Exits from the ventral cord of an unstained DE1 and a stained VI (VI-6) commissure, stained processes in the ventral nerve cord posterior to the commissures, and the stained cell body (filled arrowhead) of the VI neuron. The very thin unstained commissurc (arrow) is from a sensory neuron whose cell body is located in the lateral line (P. Sithigorngul, C. D. Johnson, and A. O. W. Stretton, unpublished observations). $B$, Exits from the ventral nerve cord of an unstained DE1

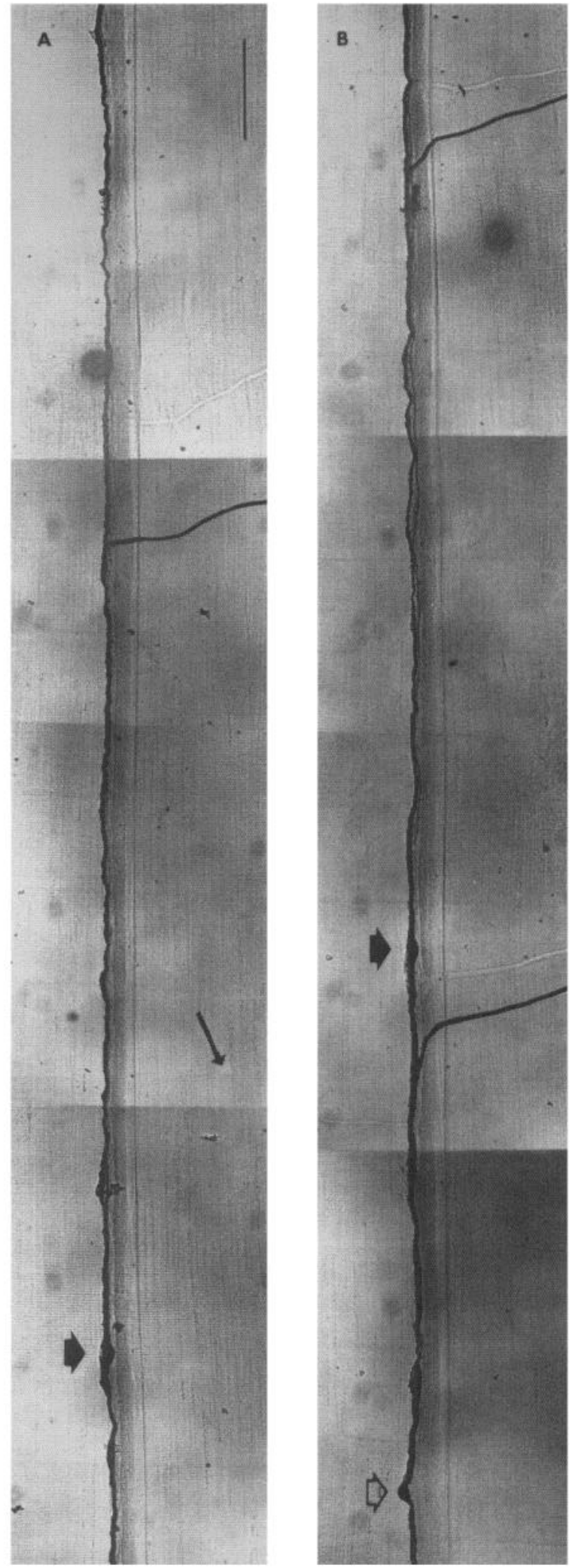

and a stained VI (VI-5) commissure near the top of the photo, and exits of unstained DE2 and DE3 and of a stained DI (DI-3) commissure near the bottom. It also shows ventral cord processes and the cell bodies of the VI (filled arrowhead) and DI (open arrowhead) neurons. Preparations were mounted, photographed, and the negative printed as described in Figure 2. The location of cell bodies from individual motor neurons is known from fiber tracing of serial sections (Stretton et al., 1978). Scale bar, $0.5 \mathrm{~mm}$. 


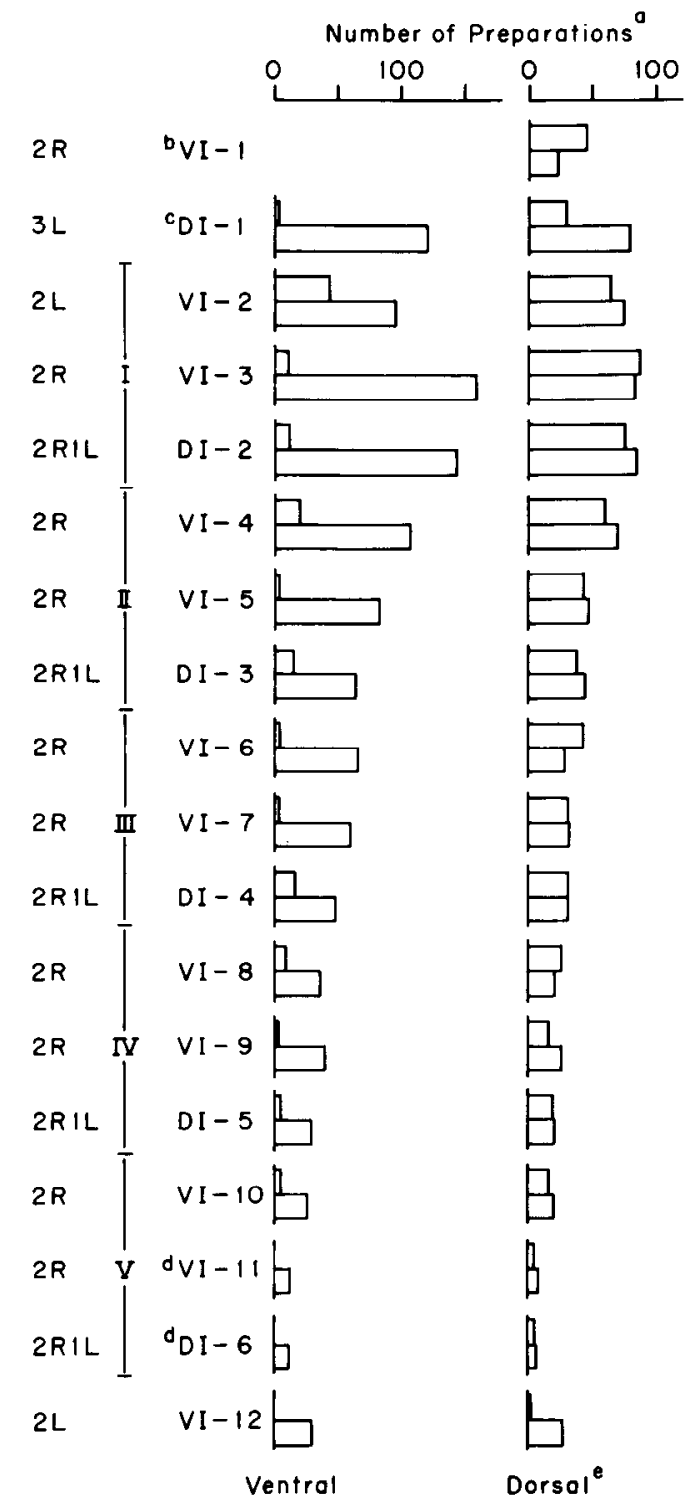

Figure 5. Graphical representation of the relative anterior/posterior positions of stained commissures. Commissure groups that contain a stained commissure are listed in anterior-to-posterior order in the first column: $2 R$, right-handed pair; $2 L$ and $3 L$, left-handed groups containing 2 and 3 commissures, respectively; $2 R 1 L$, groups containing 2 right-handed and 1 left-handed commissure. The extent of the 5 repeating units is indicated to the right of the group names (roman numerals). The stained inhibitory motor neuron is listed in the second column. The length of the bars indicates the number of preparations in which the stained commissure was anterior (upper bar of each pair) or posterior (lower bar) to the unstained commissure. Positions in ventral hypodermis and dorsal hypodermis are graphed in separate columns. Note that the stained commissure was generally posterior in ventral hypodermis and, in most cases, was anterior or posterior with nearly equal frequencies in the dorsal hypodermis. $a$. A small number of preparations in which the commissures crossed within the ventral or dorsal hypodermis (less than $5 \%$ of the total) were not included. $b$, This $2 \mathrm{R}$ group cannot be visualized in the ventral hypodermis owing to its unusual location (see text). $c$, The position of the stained DI-1 commissure was determined relative to the thicker of the 2 unstained commissures in the $3 \mathrm{~L}$ group (a DE2). It is also generally posterior to the thinner unstained commissure (a DE3) as well. $d$, The right-handed commissures in the second $2 \mathrm{R}$ and the $2 \mathrm{R} 1 \mathrm{~L}$ of the fifth repeating unit often overlap. Only preparations in which the 2 stained commissures (VI-11 and DI-6) could be distinguished on the basis of the shape of their entrances into the dorsal cord (see Fig. 8 and Table 1) were included.

\begin{tabular}{|c|c|c|c|c|}
\hline \multirow[b]{2}{*}{$\begin{array}{l}\text { Inhibitory } \\
\text { motor } \\
\text { neuron }\end{array}$} & \multirow[b]{2}{*}{$\begin{array}{l}\text { No. of } \\
\text { preparations } \\
\text { scored }\end{array}$} & \multicolumn{3}{|c|}{ Location of branch point } \\
\hline & & $\begin{array}{l}\text { Neuronal } \\
\text { bundle in } \\
\text { nerve cord } \\
(\%)\end{array}$ & $\begin{array}{l}\text { Hypo- } \\
\text { dermal } \\
\text { chalice } \\
(\%)\end{array}$ & $\begin{array}{l}\text { Lateral } \\
\text { hypo- } \\
\text { dermis } \\
(\%)\end{array}$ \\
\hline VI-1 & 81 & 90 & 4 & $6^{a}$ \\
\hline DI-1 & 92 & 100 & 0 & 0 \\
\hline VI-2 & 104 & 76 & 15 & 9 \\
\hline VI-3 & 129 & 65 & 26 & 9 \\
\hline DI-2 & 117 & 99 & 1 & 0 \\
\hline VI-4 & 90 & 50 & 39 & 11 \\
\hline VI-5 & 64 & 20 & 48 & 32 \\
\hline DI-3 & 65 & 98 & 0 & 2 \\
\hline VI-6 & 49 & 14 & 35 & 51 \\
\hline VI-7 & 52 & 8 & 33 & 60 \\
\hline DI-4 & 48 & 92 & 6 & 2 \\
\hline VI-8 & 36 & 8 & 36 & 56 \\
\hline VI-9 & 33 & 6 & 36 & 58 \\
\hline DI-5 & 32 & 91 & 9 & 0 \\
\hline VI-10 & 24 & 17 & 21 & 63 \\
\hline VI-11 & 20 & 20 & 30 & 50 \\
\hline DI-6 & 19 & 95 & 5 & 0 \\
\hline VI-12 & 28 & 4 & 10 & 86 \\
\hline VI-13 & 19 & 63 & 37 & 0 \\
\hline
\end{tabular}

In 2 preparations, the VI-1 commissure split within its sublateral branch such that two dorsal processes emerged from the branch. In 2 other preparations, the split occurred within the lateral line, ventral to the sublateral branch. In these preparations, the 2 dorsal processes possessed separate sublateral branches.

hypodermal tissue that, following fixation with glutaraldehyde, may exclude the antibodies. Postfixation treatment with pronase for $1 \mathrm{hr}$ was used in these experiments, since it was observed to increase staining of fibers and especially cell bodies in the nerve cords. Exposure to higher concentrations of pronase or for longer durations caused a decline in staining.

In the case of the VI neurons, the distance from the cell body to the commissure varies systematically along the length of the animal (Fig. 7). Measured as a fraction of the total worm length, the distance is about 0.01 in the first repeat, but then increases dramatically to 0.07 at the end of the fifth repeat and to 0.15 for VI-12. By contrast, for the $6 \mathrm{DI}$ neurons the distance is constant (about 0.01 in each case). The gradual increase in the length of the VI process means that the repeating pattern in the order of cell bodies in the ventral cord changes down the length of the animal. Thus, in the first and second repeating units, the cell body of the second VI commissure in each unit is anterior to the DE2 and DI commissures, whereas in the fourth and fifth it is posterior. In the third repeating unit, the order is variable: In some preparations the VI cell body is anterior to the cell body and commissure of DE2; in others it is posterior. Variability in the order of analogous motor neuron cell bodies has previously been noted in C. elegans (Sulston, 1976).

\footnotetext{
$e$, Stained commissures that split into several processes within the dorsal hypodermis (Fig. 6, sections 7 and 10, and Fig. 8) were scored as anterior or posterior to the unstained commissure on the basis of position prior to the branching.
} 


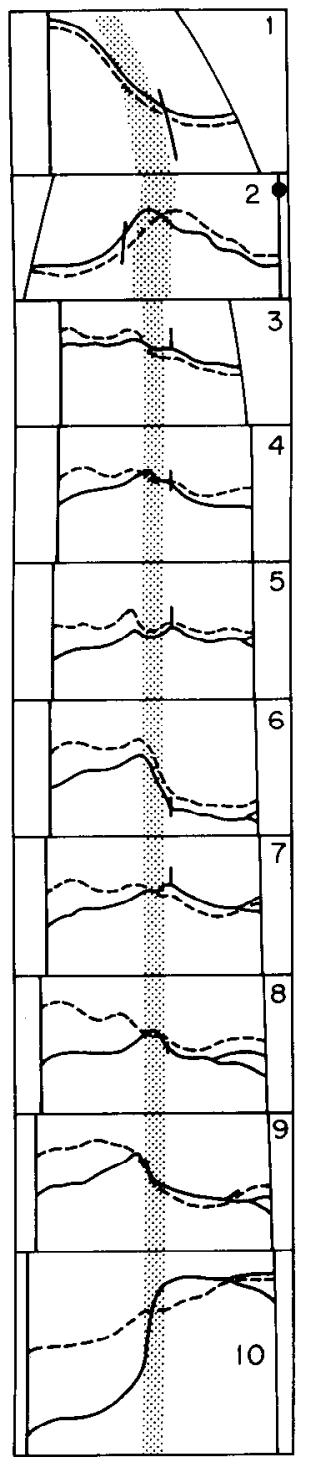

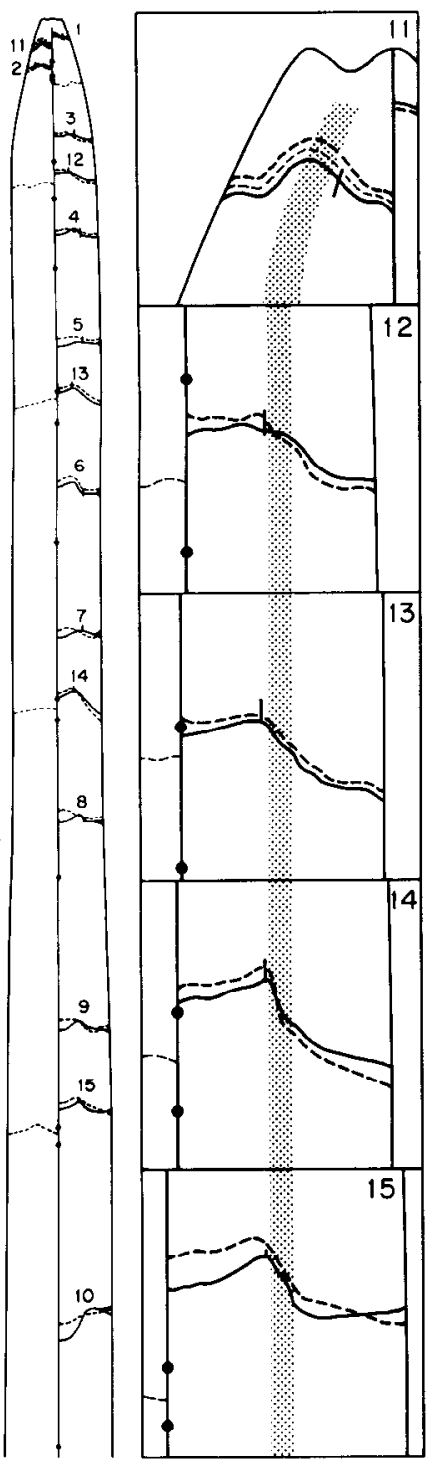
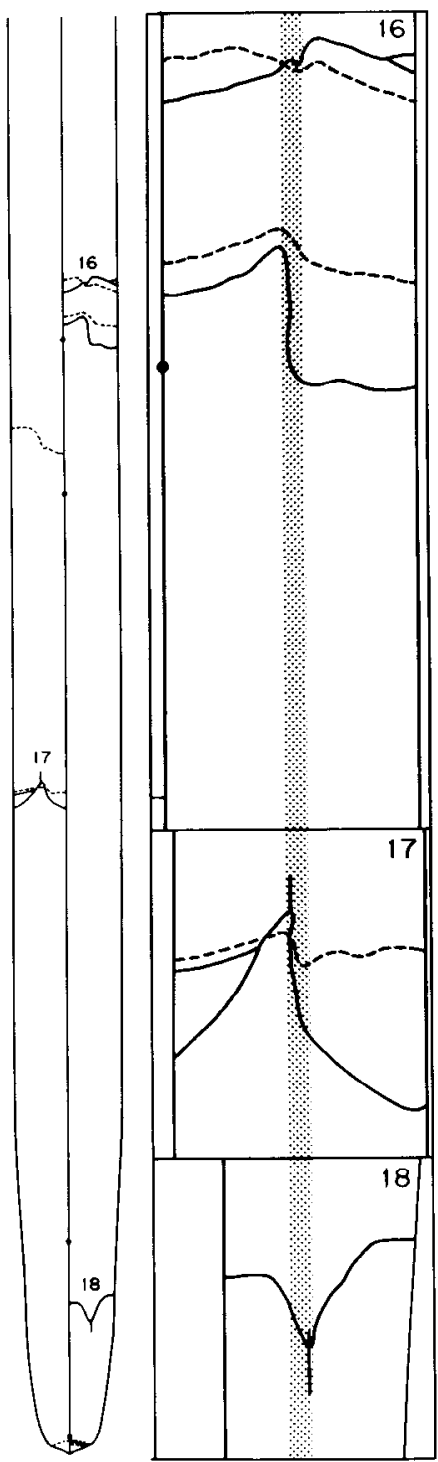

Figure 6. Diagram of the pattern of stained and unstained commissures. Sections 2 and 4, The complete set of stained (solid lines) and unstained (dashed lines) motor neuron commissures. The commissures are drawn on a tracing of the shape of a single 25$\mathrm{cm}$-long animal, cut open along the dorsal cord; the central line represents the ventral cord. Commissures were located on the diagram according to average positions, measured as a fraction of the total worm length in 26 female worms. The individual, stained commissures were traced from preparations in which the shape of the commissure was judged typical from a comparison of 20-50 examples of each cell. Unstained commissures were traced from the same preparations. The dots in the ventral cord represent the positions of the 19 stained cell bodies, which correspond to the 19 stained commissures. Cell bodies were located on the basis of averaged measurements of their positions relative to the flanking stained commissures in 5-35 preparations. Peripheral drawings (numbered to correspond with labels on central diagrams) show individual groups of commissures in more detail. The first section shows commissure groups located in the anterior half of the animal, containing a VI commissure; the third section shows the groups in the anterior half of the animal that contain a DI commissurc. Commissure groups in the posterior half of the animal are shown in the last section. Note that peripheral drawings 1,2 , and 11 are magnified $10 \times$ and the remainder $5 \times$ relative to the second and fourth sections. The position and shape of the sublateral or lateral branch drawn on some of the commissures were chosen as typical, from the data of Figure 11.

\section{Morphological features of inhibitory motor neuron commissures}

Another morphological characteristic that distinguishes the inhibitory from the excitatory motor neurons is that the commissures of inhibitors connect to both anteriorly and posteriorly directed processes in the dorsal nerve cord, whereas excitors have dorsal processes in only 1 direction (Fig. 1). In many instances (e.g., Fig. $8 A$ ) the stained commissure enters the cord and comes very close to the bundle of other neurons in the dorsal cord before branching into anterior and posterior processes. In some preparations, however, the branching occurs as the commissure approaches the dorsal nerve cord, either within the dorsal hypodermal chalice (Fig. $8 B$ ) or within the lateral hypodermis that underlies the muscle cells (Fig. 8C). Although the commissure generally splits into only 2 processes, additional branches are often seen close to the nerve cord (Fig. $8 B$ ), and examples of more complex patterns are also observed (Fig. 8, $D, E)$. The number of preparations in which particular motor neuron commissures were observed as branching near the nerve bundle in the chalice or in the lateral hypodermis is recorded in Table 1 . We can readily derive 2 generalizations. First, nearly all split commissures are from VI neurons; the DI commissures usually enter the dorsal cord unbranched. Second, the tendency of VI commissures to split increases down the length of the animal. Further, the branch points of more posterior VI commissures are more likely to occur in the lateral hypodermis than in the chalice (Table 1), and at increasing distances from the dorsal cord (data not shown). Finally, we have observed that many of the commissures that branch in the lateral hypodermis do so where the (unstained) excitatory commissure crosses the VI commissure (e.g., Fig. 6, sections 7 and 10). In a smaller number of preparations, the VI commissurc displaycd a short branch where it crossed the excitatory commissure, but this branch ended before reaching the dorsal cord (e.g., Fig. 6, section 9).

One unexpected morphological feature of the inhibitory motor neuron commissures that was revealed by these experiments was the presence on some commissures of a small longitudinal branch that occurs at one of the sublateral or lateral nerve cords 


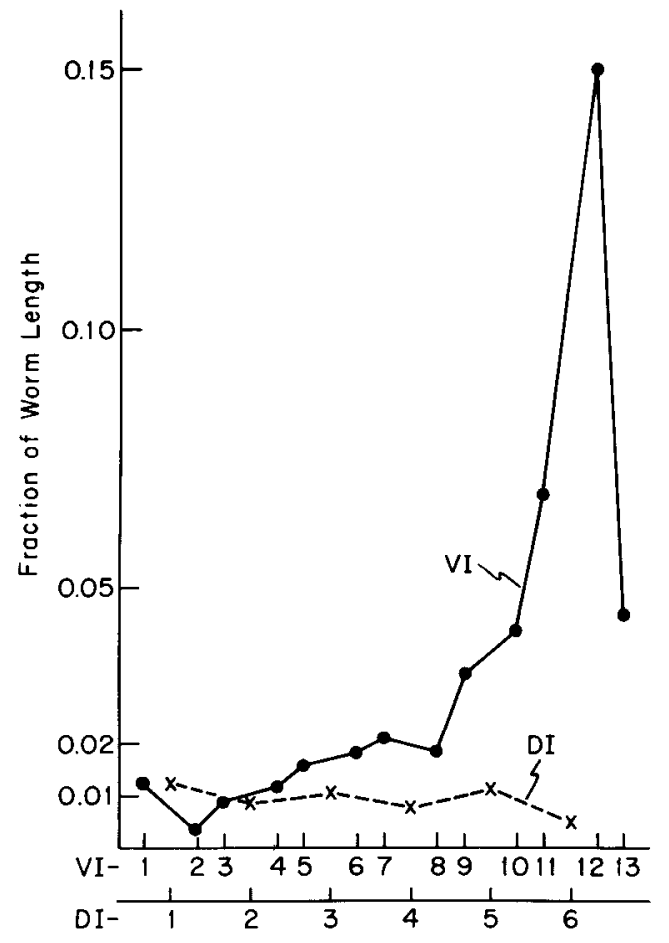

Figure 7. Graph showing distance in the ventral cord between the commissure and cell body of individual inhibitory motor neurons: the VI motor neurons (solid line), DI motor neurons (dashed line). Horizontal axis lists the neurons in anterior-to-posterior order. Distance between commissure and cell body was measured as a fraction of total worm length from the data in Figure 6.

(Fig. 10). The positions of these minor nerve cords within cross sections of an adult Ascaris are shown in Figure 9. The 4 sublateral nerve cords are found only in the front part of the animal; they are located in small hypodermal swellings, $0.1-0.2 \mathrm{~mm}$ dorsally and ventrally from the 2 lateral lines. Each sublateral cord has 4-5 intrinsic fibers, somc of which appear to be motor neurons since they make synapses to muscle cells. Although each of the motor neuron commissures crosses both a ventral and a dorsal sublateral nerve cord, we have found that sublateral branches from VI motor neuron commissures occur at the dorsal sublateral nerve cord (Fig. 10, $A, C, E, F, I$ ), whereas branches of the DI neurons occur at the ventral sublateral nerve cord (Fig. $10, B, G$ ). This is a highly reproducible feature (Table 2 ). In total, we have observed on DI commissures 404 ventral sublateral branches but only 1 (small) dorsal sublateral branch. On VI commissures we have observed 821 dorsal sublateral branches and 6 ventral lateral branches; 3 of the latter were small; the others appeared normal. Four of these exceptions occurred on the commissures of VI-2 neurons. Another unusual feature of the VI-2 commissure is that in nearly one-fifth of the preparations, this commissure displayed one or more isolated longitudinal spines as it traversed the ventrolateral hypodermis from ventral nerve cord to the lateral line (Table 2; Fig. 10D). Similar branches were observed on other commissures, especially those of VI-12 and VI-13 (Table 2; Fig. 10M). It seems possible that some of the instances in which the VI-2 commissure displayed ventral sublateral branches may actually represent such a lateral spine occurring at the position of the sublateral nerve cord, and not a true violation of the sublateral branch rule. On the other hand, the ventral sublateral branch shown in Figure $10 \mathrm{H}$, which occurs on a VI-5 neuron, appears to be a
Table 2. Small branches of commissures

\begin{tabular}{|c|c|c|c|c|c|c|c|}
\hline \multirow{3}{*}{$\begin{array}{l}\text { Inhibi- } \\
\text { tory } \\
\text { motor } \\
\text { neuron }\end{array}$} & \multirow{3}{*}{$\begin{array}{l}\text { No } \\
\text { branch }\end{array}$} & \multicolumn{4}{|c|}{ Branches in nerve cords } & \multicolumn{2}{|c|}{$\begin{array}{l}\text { Branches in } \\
\text { lateral } \\
\text { hypodermi }\end{array}$} \\
\hline & & \multicolumn{2}{|c|}{ Sublateral cord } & \multicolumn{2}{|c|}{ Lateral cord } & \multirow{2}{*}{$\begin{array}{l}\text { Ven- } \\
\text { tral }\end{array}$} & \multirow{2}{*}{$\begin{array}{l}\text { Dor } \\
\text { sal }\end{array}$} \\
\hline & & Ventra & 1 Dorsal & Ventr & Dorsal & & \\
\hline VI-1 & 0 & 0 & 115 & NA & 0 & 0 & 3 \\
\hline DI-1 & 1 & 134 & 1 & NA & 0 & 0 & 2 \\
\hline VI-2 & 2 & 4 & 184 & NA & 0 & 41 & 0 \\
\hline VI-3 & 0 & 0 & 192 & NA & 13 & 5 & 0 \\
\hline DI-2 & 0 & 192 & 0 & NA & 0 & 2 & 0 \\
\hline VI-4 & 0 & 1 & 132 & NA & 0 & 4 & 1 \\
\hline VI-5 & 0 & 1 & 69 & NA & 2 & 0 & 2 \\
\hline DI-3 & 0 & 47 & 0 & NA & 0 & 0 & 0 \\
\hline VI-6 & 0 & 0 & 58 & NA & 0 & 2 & 1 \\
\hline VI-7 & 2 & 0 & 40 & NA & 0 & 0 & 3 \\
\hline DI-4 & 6 & 31 & 0 & NA & 0 & 0 & 0 \\
\hline VI-8 & 15 & 0 & 18 & NA & 1 & 1 & 0 \\
\hline VI-9 & 27 & 0 & 8 & NA & 2 & 0 & 1 \\
\hline DI-5 & 40 & 6 & 0 & NA & 0 & 0 & 0 \\
\hline VI-10 & 30 & NA & NA & 0 & $4^{b}$ & 0 & 0 \\
\hline VI-11 & 22 & NA & NA & 0 & $1^{b}$ & 1 & 0 \\
\hline DI-6 & 22 & NA & NA & $1^{b}$ & 0 & 0 & 0 \\
\hline VI-12 & $12^{a}$ & $\mathrm{NA}$ & NA & 7 & 20 & 0 & 2 \\
\hline VI-13 & $14^{\prime}$ & NA & NA & 32 & 49 & 8 & 11 \\
\hline
\end{tabular}

This table records the number of preparations in which small branches were observed in a sublateral nerve cord, in a lateral nerve cord, or unassociated with other neural fibers in the lateral hypodermis. NA, Relevant nerve cord is not present (i.e., posterior to the fourth repeating unit for the sublateral nerve cords and anterior to the fifth repeating unit for the ventral lateral nerve cord).

${ }^{a}$ In most of these preparations the commissure runs along the lateral nerve cords although no branches were observed.

${ }^{b}$ These branches occur at the margin of the lateral line. We have assigned them to the lateral nerve cord since, in most preparations, the sublateral cords end anterior to this commissure (it is possible that in these preparations the sublateral nerve cord is abnormally long).

true exception. It is important to note that the rules governing the occurrence of sublateral branches apply not only to the usual right-handed commissures, but to left-handed DI and VI motor neuron commissures as well. This is shown both from the position of branches on the commissures of the DI-1 (Fig. 10B) and VI-2 (Fig. 10C) motor neurons - which routinely traverse the animal's left side-and from the appropriate positioning of branches on those rare commissures which display a reversed handedness (data not shown).

The sublateral branches of the inhibitory motor neuron commissures can extend both anteriorly and/or posteriorly (Fig. 10I) from the commissure. They often have a complex fine structure. The anterior and posterior length of commissure branches, measured from well-stained preparations, is illustrated in Figure 11. Branches $200-500 \mu \mathrm{m}$ long are a prominent feature of all inhibitory motor neuron commissures located in front of the fourth repeating unit. For some commissures, there is a clear bias toward anteriorly projecting branches. These include all of the stained commissures in the first 3 repeats, with the exception of VI-2 (and possibly VI-6). Branches of VI-2 usually extend in both directions. The DI- 1 commissure has short (less than 200 $\mu \mathrm{m})$, generally posterior branches. Finally, the VI-1 commissure has moderately long (200-400 $\mu \mathrm{m})$ branches that project in both directions. Short branches are sometimes observed on commissures in the fourth repeating unit; commissures posterior to this have no sublateral branches (Table 2). This pattern most 


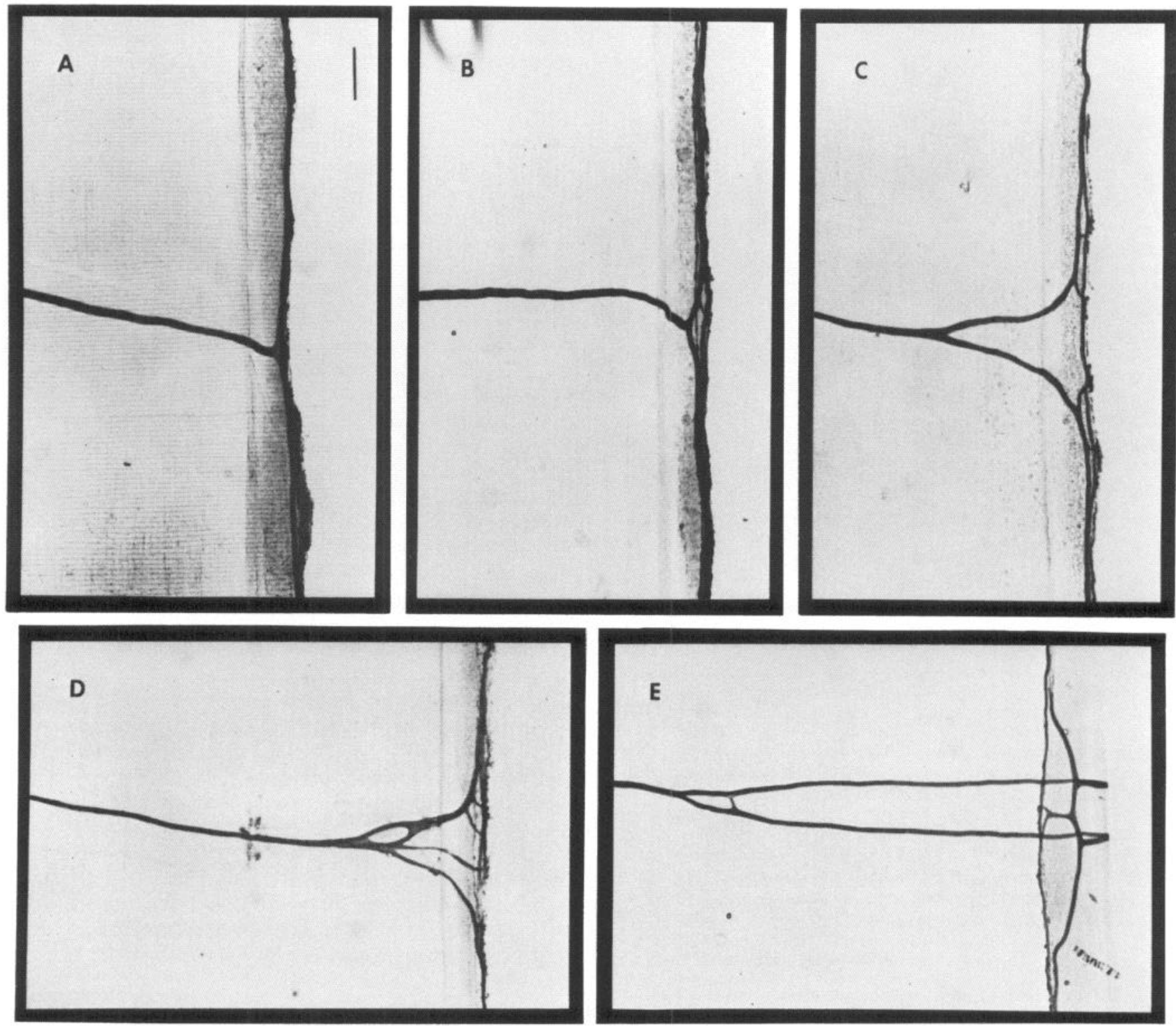

Figure 8. Photographs showing commissures approaching and entering the dorsal nerve cord. $A$, Unbranched commissure entrance of the dorsal nerve cord from DI-4 motor neuron. Unbranched entrances are typical of all DI commissures and of VI commissures in the anterior region of the animal. $B$, Commissure branched in the dorsal hypodermal chalice from a VI-5 motor neuron. $C$, Commissure branched in the dorsal hypodermis from a VI-8 motor neuron. Branched commissures are typical of VI motor neurons in the posterior region of the animal. $D$ and $E$, More complex branching of commissures seen on a VI-8 $(D)$ and a VI-6 $(E)$ motor neuron. In $E$, the dorsal hypodermal chalice is folded to the left so that it appears that the dorsal cord lies on top of the commissure. These photographs were taken with a $2.5 \times$ objective using illumination from a standard bright-field condenser. The film was developed in $\mathrm{HC1} 10$ developer (dilution D for $8 \mathrm{~min}$ ) and printed without the use of polycontrast filters. These procedures minimize the artifactual density in the hypodermis; however, unstained commissures are generally not visible on the prints. Scale bar, $0.1 \mathrm{~mm}$.

likely reflects the decline in fiber diameter and eventual termination of the sublateral nerve cords near the end of the fourth repeating unit (Stretton, 1976).

The 2 most posterior stained commissures generally have prominent lateral branches (Fig. 10, $L, M$; Fig. 11). These branches are associated not with sublateral cords, which end anterior to these commissures, but with the lateral nerve cords (Table 2). The 4 lateral nerve cords are located at dorsal and ventral margins of the 2 lateral lines (Fig. 9). Anterior to the fifth repeating unit, the ventral lateral nerve cords end; the dorsal lateral cords project forward to the nerve ring in the head. The VI-13 commissure has relatively long, generally posterior branches in the right dorsal lateral nerve cord (Figs. 10M and
11); those of VI-12 are generally anterior in the left dorsal lateral nerve cord (Figs. $10 L$ and 11). These commissures often also display shorter branches in their respective ventral lateral nerve cords (e.g., Fig. 10M). Short branches in the lateral nerve cord are also occasionally observed on more anterior commissures (Table 2; Fig. 10E).

\section{Discussion}

Nematodes have long been recognized as excellent material for the study of neuroanatomy. Classical studies of Ascaris by light microscopy, culminating with those of Hesse (1892), who first described the commissures, and Goldschmidt (1908), emphasized the small cell number and relative simplicity of the mor- 


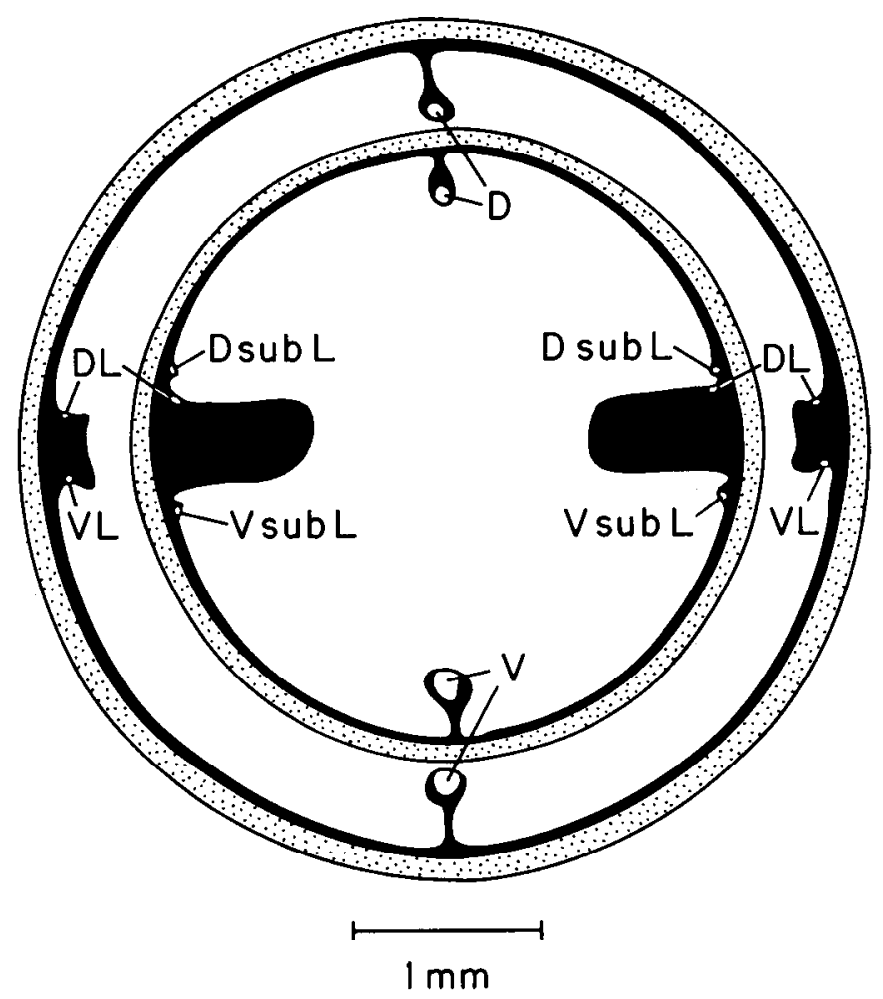

Figure 9. Locations of major and minor longitudinal nerve cords. This diagram was traced from photographs of 2 cross sections of an adult Ascaris. Only the cuticle (stippled), hypodermis (black), and nerve cords embedded in the hypodermis were traced (muscle, intestine, and gonads were not drawn), and the cross section from the anterior region of the animal (which is narrower) was drawn inside of the cross section from the posterior region. The major ventral $(V)$ and dorsal $(D)$ nerve cords are located at the inner edge of medial swellings of hypodermis. The ventral sublateral $\left(V_{s u b L}\right)$ and dorsal sublateral $(D s u b L)$ nerve cords are located in small hypodermal swellings that are adjacent to the large lateral hypodermal swellings referred to as lateral lines. The sublateral cords are present only in the anterior region of the animal. The ventral lateral $(V L)$ and dorsal lateral $(D L)$ nerve cords are located at the margins of the lateral lines. The dorsal lateral cord extends through the entire animal; the ventral lateral cord is present only in the posterior region. There is also a small lateral nerve cord, usually located centrally at the medial edge of the lateral line, which is not shown on the diagrams.

phology of neurons in these animals, as well as the constancy of the gross morphological features displayed by single cells when comparing different individuals. These observations were central to the formulation of the concept of the identified neuron, which has been so important in modern invertebrate neurobiology. In a more recent effort, the entire nervous system of the free-living nematode, Caenorhabditis elegans, has been reconstructed from electron micrographs of serial sections to produce a detailed morphological picture that includes the complete branching pattern of each of the animal's nerve cells and an extensive listing of anatomically defined synapses (see White et al., 1986). In comparing the morphology of neurons from $A s$ caris with those of $C$. elegans, it is clear that most cells display many unique anatomical characteristics which are conserved between the 2 species. Thus an identified neuron can be studied in both species.

Goldschmidt's (1908) most detailed studies of Ascaris were of the neuronal cell bodies and processes located in the ganglia surrounding the nerve ring in the head. The neuronal processes and cell bodies located in the major, ventral, and dorsal nerve cords of Ascaris have been the object of more recent anatomical studies (Stretton et al., 1978, and unpublished observations). The nerve cords contain the cell bodies and processes of motor neurons that innervate the body wall muscle cells responsible for locomotory behavior. Based on morphology, the motor neurons were divided into 7 classes (Fig. 1); sequential members of each class were arranged in a repeating pattern down the length of the animal. The present study uses antisera specific to a neurotransmitter to investigate the anatomy of motor neurons in Ascaris; we show that 19 of the 45 motor neurons with commissures are stained by GABA-specific antibodies. On the basis of our previous knowledge of motor neuron anatomy, we have concluded that the stained neurons include the $13 \mathrm{VI}$ cells and the $6 \mathrm{DI}$ cells. It is interesting to note that GABA appears to be present throughout the cell-not just in regions of the cell containing synapses. In particular, staining of the VI motor neuron commissures depends on the presence of GABA in a process that connects the ventral axon of the neuron with its dorsal dendrite-a part of the cell that has no output synapses (Stretton et al., 1978). It appears that, at least in the case of nematode inhibitory motor neurons, the neurotransmitter is not restricted to areas of the cell from which it is released. Although it seems likely that this applies as well to the GABA-synthetic enzyme, glutamic acid decarboxylase (GAD), it will be necessary to test this possibility with GAD-specific antisera or by direct enzyme assay in the future.

Our ability to carry out these experiments depended not only on the accumulated knowledge of nematode neuroanatomy, electrophysiology, and biochemistry, and the availability of specific anti-GABA sera; the protease-dissociated preparation that we have developed was important, since it allowed the visualization of both stained and unstained commissures in wholemounts from many individuals. In the present experiments, muscle cells were removed by injection with a crude preparation of bacterial collagenase. In preliminary experiments, we found that it was important not to allow this digestion to proceed too far and to fix the preparation with glutaraldehyde immediately: Overdigested preparations with visible breaks in the hypodermis generally stained poorly. Although the glutaraldehyde fixation was necessary (preparations fixed with formaldehyde alone do not stain), the fixed tissue presented a considerable barrier to penetration of antibodies. This problem was minimal where staining of commissures was concerned; these processes are embedded in the thin syncytial hypodermis that underlies the muscle cells, and the selective staining of inhibitory motor neuron commissures reported in this paper was strong and highly reproducible. Lack of penetration is a more severe problem in staining of cell bodies and processes in the nerve cords. In order to increase penetration, we included a short incubation with a concentrated pronase solution and overnight treatment with detergent before applying the primary antiserum.

The identification of specific inhibitory motor neurons is based, to a large extent, on a comparison of the present results with previous studies. Tracing of fibers from serial sections has identified all of the motor neurons anterior to the fifth repeating unit on the basis of the morphology of their processes in the ventral and dorsal nerve cords (Stretton et al., 1978, and unpublished observations). The synaptic connectivity of the motor neurons has been examined by electron-microscopic analysis of selected sections, and the regions of the cell that contain neuromuscular 

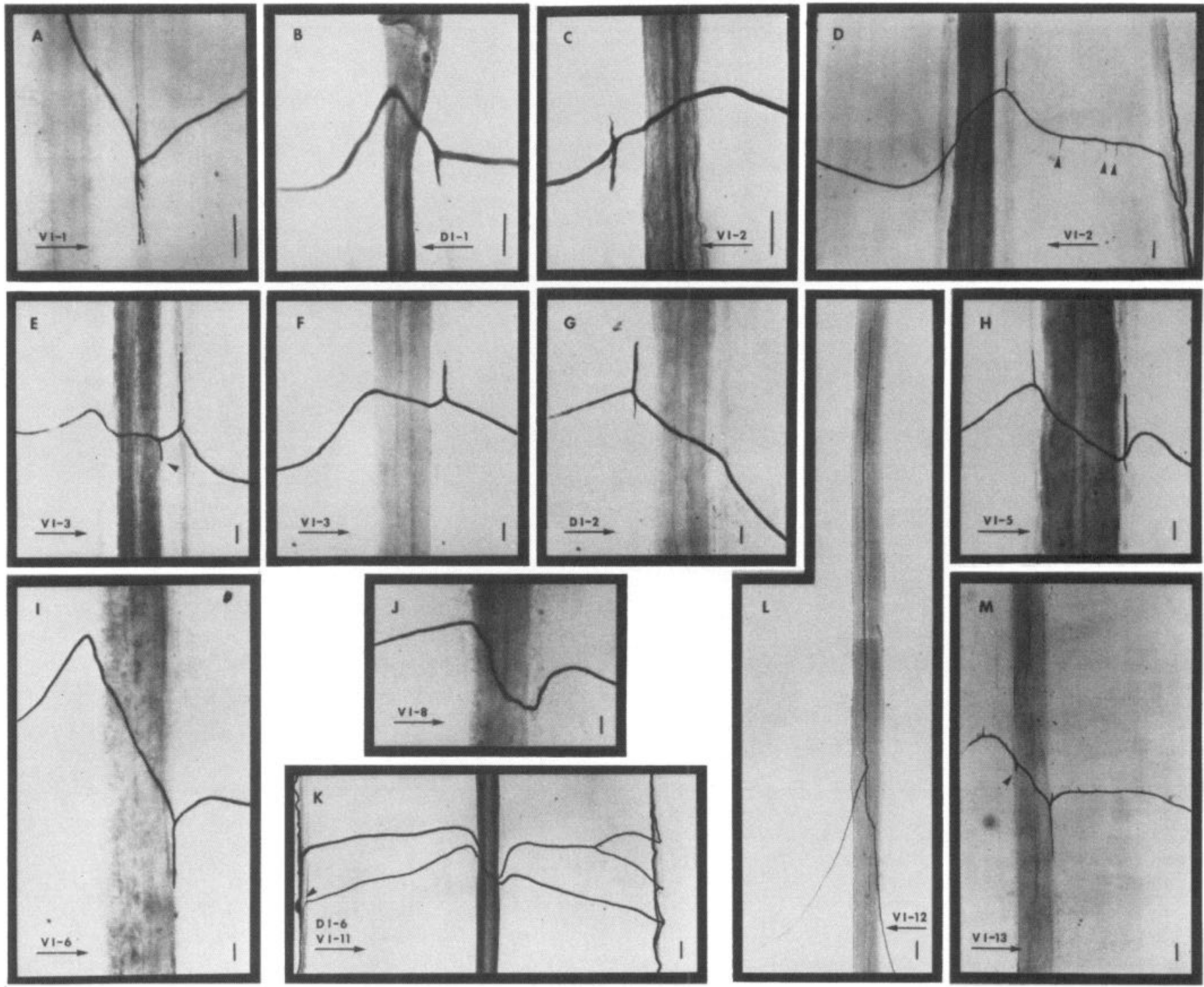

Figure 10. Collage of commissure branch photographs. Each photograph is labeled with a horizontal arrow located in ventral hypodermis, which points dorsally and underlines the name of the stained neuron. $A$, Typical VI-1 branch in right dorsal sublateral nerve cord. $B$, Typical DI-1 branch in left ventral sublateral nerve cord. $C$, Typical VI-2 branch in left dorsal sublateral nerve cord. $D$, Photograph of an unusual VI-2 commissure. In addition to the typical branch in the left dorsal sublateral nerve cord, this commissure also displays several short, posteriorly directed spines in the ventral hypodermis (arrowheads) and a branch in the ventral sublateral nerve cord. $E$, VI-3 commissure with a branch in the right dorsal sublateral nerve cord and a short branch in the right dorsal lateral nerve cord (arrowhead). F, VI-3 branch in right dorsal sublateral nerve cord. This branch is typical of VI branches on neurons VI-3 through VI-7. G, DI-2 branch in right ventral sublateral nerve cord. This branch is typical of DI branches on neurons DI-2 through DI-4. H, VI-5 commissure with branches in both the right dorsal sublateral nerve cord and the ventral sublateral nerve cord. Ventral branches of the VI motor neurons are rare. $I$, VI- 6 commissure with a posteriorly directed branch in the right dorsal sublateral nerve cord. $J$, VI-8 commissure with no sublateral branch. Commissures without branches are common on VI-8 through VI-11 and on DI-5 and DI-6 neurons. $K$, Photograph of VI-11 and DI-6 commissures. On the ventral side, the DI commissure is more anterior and relatively thicker. The commissures cross in the lateral line so that on the dorsal side the VI commissure, which splits as it approaches the dorsal cord, is more anterior. Note also the DI cell body in the ventral nerve cord (arrowhead). These commissures typically have no sublateral or lateral branches. $L$, VI-12 branch in the left dorsal lateral nerve cord. In this part of the animal, the lateral nerve cords are located within the lateral line. $M$, VI13 branch in the right dorsal lateral nerve cord. This commissure also displays a short branch in the right ventral lateral nerve cord (arrowhead) and a number of small spines in ventral and dorsal hypodermis. Photographs for $A, B$, and $C$ were taken through a $6.3 \times$ objective. The remainder of the photographs were taken through a $2.5 \times$ objective. Film development and printing were as described in Figure 8 . Scale bars, $0.1 \mathrm{~mm}$.

synapses were determined. These experiments have, to date, been extended through the third repeating unit (J. Donmoyer, P. Desnoyers, and A. O. W. Stretton, unpublished observations). Electrophysiological techniques have been applied in analyzing the site of neuromuscular output and the physiological sign of 29 of the motor neurons with commissures, including several members of each of the 5 classes (Stretton et al., 1978; Walrond et al., 1985; R. Davis, P. Hanrahan, and A. O. W. Stretton, unpublished observations). All members of the VI and DI classes examined were shown to be inhibitory motor neurons, i.e., their stimulation causes hyperpolarization of ventral or dorsal muscle cells, respectively. The results of the experiments reported here are fully consistent with the anatomical and physiological characterizations: Each of the known inhibitory motor neurons, 

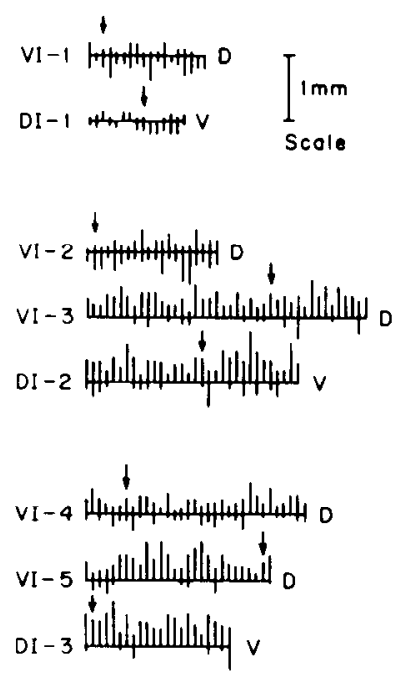
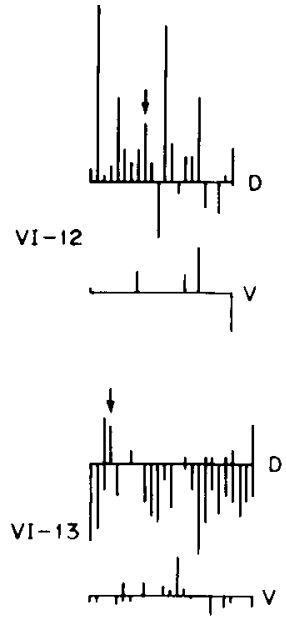

inhibitory motor neurons. These cells have been assigned to either the VI or DI class according to the morphological features that are consistently present on the fully characterized neurons. These featurcs include the position of the motor neuron's cell body relative to its commissure, the propensity of VI, but not DI, commissures to branch as they approach the dorsal cord, and the presence of branches at specific sublateral and lateral nerve cords. For example, the VI-1 has been studied with anatomical, but not with electrophysiological, techniques. It has been classified as a VI cell on the basis of its gross morphology, the location of its neuromuscular synapses, and its synaptic connectivity in the ventral and dorsal nerve cords. The presence on the VI-1 commissure of a prominent branch at the dorsal sublateral nerve cord is consistent with this conclusion: Nearly all sublateral branches on fully characterized VI cells are in the dorsal sublateral cord, whereas those of the DI cells are in the ventral sublateral cord. Furthermore, the VI- 1 commissure is occasionally split as it approaches the dorsal cord, whereas the DI commissure rarely displays this feature.

Neurons associated with commissures in the fifth repeating unit have not been studied with electrophysiological techniques. Biochemical studies of the localization of ChAT (Johnson and Stretton, 1985) revealed that one of the commissures in each of the right-handed pairs, as well as the single commissure to the left, contained the enzyme. These cells were assigned to $l$ of the 3 excitatory motor neuron classes because of the analogous position of their commissures in the repeating unit to fully characterized excitatory motor neurons in the more anterior repeats. The present data indicate that the commissures without ChAT stain with the anti-GABA serum. The stained commissures are called VI-10, VI-11, and DI-6 in a continuation of the repeating pattern. Consistent with this classification, the cell bodies of the 2 VI cells are a long distance from their commissures, whereas the cell body-commissure distance is short for the DI cell. Furthermore, the VI commissures often branch before reaching the dorsal nerve cord, whereas the DI commissure generally does not. This classification is consistent with a preliminary analysis of the morphology of these neurons from serial sections $(M$. Halloran, J. Donmoyer, and A. O. W. Stretton, unpublished observations).

Commissures posterior to the fifth repeating unit have not been examined with either electrophysiological or anatomical techniques. In biochemical experiments, localization of ChAT was observed in the anterior thick member of the group of 2 left-handed commissures (Johnson and Stretton, 1985); the posterior thin member of this group is stained with the anti-GABA antiserum. Based on the long distance posterior to its cell body in the ventral cord, the prominence of its branches in the dorsal lateral nerve cord as compared to those in the ventral lateral nerve cord, and the frequent occurrence of split commissures, we believe that this commissure is from motor neuron VI- 12 . Finally, the single right-handed commissure in the tail has been classified as VI-13 using similar criteria.

Cells that are morphologically analogous to the Ascaris motor neurons are found in C. elegans (White et al., 1976, 1987). The number, arrangement, and morphology of cells are nearly identical for the 2 species (see Stretton et al., 1985); in C. elegans, there are exactly 13 and 6 cells, respectively, in 2 classes of motor neurons that are morphologically analogous to the VI and DI (called VD and DD, respectively; White et al., 1976, 1987). In addition, E. Hedgecock (personal communication) and S. McIntire and H. R. Horvitz (personal communication) have

Neurons that label with anti-GABA serum but have not been studied with electrophysiological techniques are presumptive 
concluded, from an analysis of stained cell bodies in the ventral cord and of stained commissures, that all of the VD and DD neurons are stained by anti-GABA serum in $C$. elegans. Thus, in this case at least, neurotransmitter phenotype as well as the morphological features of neurons appears to be conserved between species.

One significant morphological feature of the inhibitory motor neurons that was discovered during the course of these experiments is the occurrence of small branches in a specific subset of the minor nerve cords that are embedded in or located next to the lateral lines. Branches that occur at one of the sublateral nerve cords are a highly reproducible feature of the inhibitory motor neurons located anterior to the fourth repeating unit. A similar bias appears to operate in commissures of VI-12 and VI-13, even though the branches of these commissures occur not in the sublateral but in the lateral nerve cords. The occurrence and position of sublateral and lateral branches thus provide morphological criteria that distinguish between the 2 classes of inhibitory cells. Further, detailed analysis of the morphology of the branches shows that there are distinguishing features between individual neurons within the 2 classes as well.

Although the function of the sublateral branches has not yet been investigated in detail, preliminary electrophysiological and electron-microscopic analysis suggests that some of the branches may receive input from excitatory neurons located in the sublateral nerve cords (J. D. Angstadt, J. E. Donmoyer, and A. O. W. Stretton, unpublished observations). No evidence for output synapses has been obtained. Perhaps sublateral branches are important during the movements of the anterior part of the animal occurring in the right-left plane. During such behavior, activation of excitatory motor neurons in the left sublateral nerve cords could not only cause contraction of those muscle cells that send arms to the sublateral nerve cords, but cause relaxation of ventral and dorsal muscle cells by activating the VI-2 and DI-1 motor neurons. Similarly activation of excitatory molor neurons in the right sublateral nerve cords would directly stimulate innervated muscle cells and cause ventral and dorsal relaxation by activation of the VI-1, VI-3, and DI-2 in the head. That these interactions may be less significant in more posterior regions of the animal is indicated by the decline in the number of muscle cells that send a branch to the sublateral nerve cord (Stretton, 1976), by the observation that movement in the rightleft plane is restricted to the anterior part of the animal, and by the present data, which show that left-handed inhibitory commissures with branches in the sublateral nerve cords arc rcstricted to the head.

Prominent sublateral branches have not been observed in $C$. elegans, although there are varicosities in the commissures where they cross the sublateral cords (S. McIntire and H. R. Horvitz, personal communication). It is interesting to note that the DI-1 and VI-2 neurons in C. elegans are also left-handed and that the other unusual details of commissure handedness in the head are also conserved (except that the DE1, which is paired with VI-2, goes to the right). On the other hand, the left-handed DE1/ VI commissure pair posterior to the fifth repeating unit of $A s$ caris is right-handed in $C$. elegans and 2 of the DE2 neurons that are right-handed in Ascaris are left-handed in C. elegans. Unusual commissure direction in the body has not been conserved. These observations are consistent with a functional role for reversed commissures in the head.

The function of the commissure branches observed in the lateral nerve cord on VI-12 and VI-13, and occasionally on more anterior commissures, is even less clear. In $C$. elegans these nerve cords contain the fibers of touch-sensitive sensory neurons, called ALM and PLM, as well as the fiber of associated neurons called ALN and PLN, respectively (Chalfie and Sulston, 1981; White et al., 1986). PLM and PLN processes are in the ventral lateral nerve cord, ALM and ALN in the dorsal lateral nerve cord. The cell bodies of PLM, PLN, and ALN are located in the tail. The ALM cell bodies are located (in Ascaris) in the lateral line at about the position of the DE2/DI pair in the first repeat (P. Sithigorngul, C. D. Johnson, and A. O. W. Stretton, unpublished observations). It is possible that the inhibitory motor neurons might receive input from neurons in the lateral cords and that this information might be important in the control of locomotion.

\section{References}

Chalfie, M., and J. Sulston (1981) Developmental genetics of the mechanosensory neurons in Caenorhabditis elegans. Dev. Biol. 82: 358370 .

del Castillo, J., T. A. Morales, and V. Sanchez (1963) Action of piperazine on the neuromuscular system of Ascaris lumbricoides. Nature 200: 706-707.

del Castillo, J., W. C. de Mello, and T. Morales (1964a) Mechanism of the paralyzing action of piperazine on Ascaris muscle. Br. J. Pharmacol. 22: 463-477.

del Castillo, J., W. C. de Mello, and T. Morales (1964b) Inhibitory action of $\gamma$-aminobutyric acid (GABA) on Ascaris muscle. Experientia 20: $141-143$.

Goldschmidt, R. (1908) Das Nervensystem von Ascaris lumbricoides und Ascaris megacephala. 1. Z. Wissenschaft. Zool. 90: 73-136.

Hesse, R. (1892) Über das Nervensystem von Ascaris megalocephala. Z. Wissenschaft. Zool. 54: 548-568.

Johnson, C. D., and A. O. W. Stretton (1985) Localization of choline acetyltransferase within identified motoneurons of the nematode $A s$ caris. J. Neurosci. 5: 1984-1992.

Martin, R. J. (1980) The effect of $\gamma$-aminobutyric acid on the input conductance and membrane potential of Ascaris muscle. Br. J. Pharmacol. 71: 99-106.

Martin, R. J. (1982) Electrophysiological effects of piperazine and diethylcarbamazine on Ascaris suum somatic muscle. Br. J. Pharmacol. 77: 255-265.

Storm-Mathisen, J., A. K. Leknes, A. T. Bore, J. L. Vaaland, P. Edminson, and O. P. Ottersen (1983) First visualization of glutamate and GABA by immunocytochemistry. Nature $301: 517-520$.

Stretton, A. O. W. (1976) Anatomy and development of the somatic musculature of the nematode Ascaris. J. Exp. Biol. 64: 773-788.

Stretton, A. O. W., R. M. Fishpool, E. Southgate, J. E. Donmoyer, J. P. Walrond, and I. S. Kass (1978) Structure and physiological activity of the motorneurons of the nematode Ascaris. Proc. Natl. Acad. Sci. USA 75: 3493-3497.

Stretton, A. O. W., R. E. Davis, J. D. Angstadt, J. E. Donmoyer, and C. D. Johnson (1985) Neural control of behavior in Ascaris. Trends Neurosci. 8: 294-300.

Sulston, J. E. (1976) Post-embryonic development in the ventral cord of Caenorhabditis elegans. Phil. Trans. R. Soc. Lond. [Biol.] 275: 287-297.

Walrond, J. P., I. S. Kass, A. O. W. Stretton, and J. E. Donmoyer (1985) Identification of excitatory and inhibitory motoneurons in the nematode Ascaris by electrophysiological techniques. J. Neurosci. 5: $1-8$.

Wenthold, R. J., J. M. 7empel, M. H. Parakkal, K. A. Reeks, and R. A. Altschuler (1986) Immunocytochemical localization of GABA in cochlear nucleus of the guinea pig. Brain Res. 380: 7-18.

White, J. G., E. Southgate, J. N. Thomson, and S. Brenner (1976) The structure of the ventral nerve cord of Caenorhabditis elegans. Phil. Trans. R. Soc. Lond. [Biol.] 275: 298-327.

White, J. G., E. Southgate, J. N. Thomson, and S. Brenner (1987) The structure of the nervous system of Caenorhabditis elegans. Phil. Trans. R. Soc. Lond. [Biol.] (in press). 